\title{
Erythrocyte Differentiation
}

National Cancer Institute

\section{Source}

National Cancer Institute. Erythrocyte Differentiation. NCI Thesaurus. Code C96833.

A biological process that refers to the maturation of erythrocytes. 